$$
\text { Conf }-9010256 \cdot-4
$$

WSRC-RP- $-89-465$

DE91 005702

\title{
ON-LINE ANALYZERS \\ TO \\ DISTRIBUTED CONTROL SYSTEM LINKING (U)
}

by

S.F. Peterson, B.R. Buchanan, and M.A. Sanders

Westinghouse Savannah River Company

Savannah River Laboratory

Aiken, South Carolina 29808

(803) $725-5251$

\author{
Westinghouse Computer Symposium \\ Fox Chapel Yacht Club \\ Fox Chapel, Pennsylvania \\ October 1990
}

\section{MASTER}

The information contained in this article was developed during the course of work under Contract No. DE-AC09-89SR18035 with the U.S. Department of Energy. By acceptance of this paper the publisher and/or recipient acknowledges the U.S. Government's right to retain a non-exclusive, royalty-free license in and to any copyright covering this paper along with the right to reproduce and to authorize others to reproduce all or part of the copyright paper.

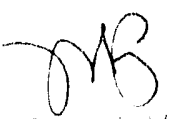


WSRC-RP-89-465

\section{ON-ILINE ANALYZER TO DISTRIBUTED CONTROL SYSTEM LINKING (U)}

by

S. F. Peterson, B. R. Buchanan, and M. A. Sanders

Westinghouse Savannah River Company

Savannah Fiver Laboratory

Aiken, Sout" Carolina 29808

A paper proposed for presentation at the

Annual Westinghouse Computer Symposium

Pittsburgh, PA

October 22-23, 1990

and for publication in the proceedings

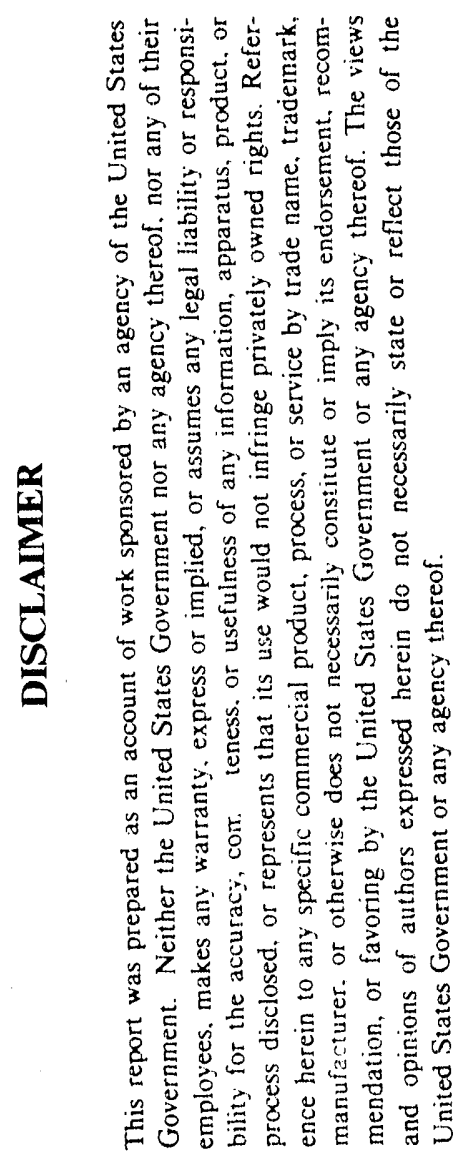

This paper was prepared in connection with work done under Contract No. DE-ACO9-89SR18035 with the U.S. Department of Energy. By acceptance of this paper, the publisher and/or recipient acknowledges the U.S. Government's right to retain a nonexclusive, royalty-free license in and to any copyright covering this paper, along with the right to reproduce and to authorize others to reproduce all or part of the copyrighted paper.

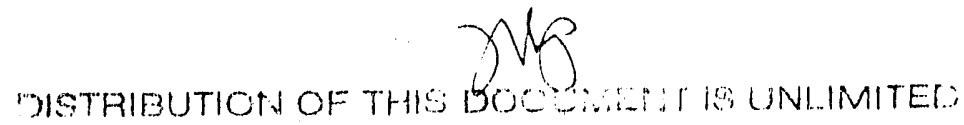


WSRC-RP-89-465

\author{
ON-LINE ANALYZERS \\ TO \\ DISTRIBUTED CONTROL SYSTEM LINKING (U) \\ by \\ S.F. Peterson, B.R. Buchanan, and M.A. Sanders \\ Westinghouse Savannah River Company \\ Savannah River Laboratory \\ Aiken, South Carolina 29808
}

\begin{abstract}
The Analytical Development Section (ADS) of the Savannah River Laboratory is developing on-line analyzers to monitor various site processes. Data from some of the online analyzers (OLA's) will be used for process control by distributed control systems (DCS's) such as the Fisher PRoVOX. A problem in the past has been an efficient and cost effective way to get analyzer data onto the DCS data highway. ADS is developing a system to accomplish the linking of OLA's to PRoVOX DCS's. The system will be described, and results of operation in a research and development environment given. Plans for the installation in the production environment will be discussed.
\end{abstract}

\title{
INTRODUCTION
}

The Analytical Development Section (ADS) of the Savannah River Laboratory (SRL) is developing on-line analyzers (OLAs) to monitor various site processes. The information from some of these analyzers is used to control chemical process operations at the Savannah River Site. Ideally, the analyzer information should be available to the Distributed Control Systems (DCSs) that monitor and control the various processes. However, interfacing such information to a DCS is not a trivial exercise. The data highway on a typical DCS is compatible only with itself. The Analytical Development Section is developing a system to accomplist, this linking of the on-line analyzers to a distributed control system. The system is being developed and demonstrated in a technical (R\&D) environment. The system consists of a Local Area Network (LAN) that interconnects a Fisher PRoVOX DCS with several on-line analyzers and office computers. This system is being used to define the required LAN hardware and software which will then be used to demonstrate information transfer from the on-line analyzers to the DCS. This information transfer will include the control of the analyzers through the DCS, and control of the monitoring and Quality Assurance (QA) functions for the analyzers from the remotely located (in offices) computers. The system will serve as a prototype for similar network installations in other site operating areas.

\section{BACKGROUND}

The Savannah River Site is a produrtion complex for the U.S. Department of Energy (DOE). The major facilities at the site are: fuel and target fabrication facilities, nuclear reactors, chemical separations plants, and a defense waste processing area. In the chemical separations facilities, irradiated materia!s from the reactors are dissolved and the desired products separated from waste materials. 
Along with many other functions, the Savannah River Laboratory provides technical support for the site operating areas. One initiative in support of the Separations Areas has been the recommendation to replace the chemical separation processes 1950s era control system with a modern, computer based control system. This effort began about 10 years ago, and substantial progress has been made. The incentives for this effort include improved safety, increased product throughput and quality, lower operating costs, and enhanced process versatility. Improvement in safety comes about through a reduction in radiation exposure to operating/analytical personnel and a reduction in the number of process incidents, many of which have serious safety potential. The increased throughput and product quality is possible by operating the process closer to optimum conditions than is possible with manual control. Eliminating or reducing recycle steps caused by deviations from desired process conditions, optimized control of reagent usage, and decreased analytical sample load will reduce operating costs. A modern distributed control system can be easily and quickly reconfigured to adapt to changing flowsheets, product specifications, starting materials, or programmatic changes.

With the advent of modern process control systems, it was soon realized that the process monitoring techniques needed to be improved. The old ways of controlling chemical process were no longer adequate. In the past samples were taken from process tanks periodically and transported to the laboratory for analysis. The turn-around time for results from these samples could be 8 hours or longer. Not an ideal information source for a fast responding, computerized process control system. Typical real-time process sensors provided only a limited amount of information to the process control engineers.

Temperature, pressure, level, and flow data give only a secondary view into the chernistry of the system. The process control environment had been limited to these sensors because the more sophisticated instrumentation required a dedicated expert to interpret the observed data. The introduction of computers to control the instruments and perform the data interpretation has provided solutions to many of these problems.

The Analytical Development Section of SRL has an ongoing program to acquire or develop and install on-line process analyzers. The objective of this program is to have site process fully monitored by on-line analyzers, with the observed analytical data interpreted and available to a control system.

To accomplish these objectives requires exptensive communications between the DCS and the OLA. Control information must be transmitted in both directions, then the analyzer output must be interpreted and transmitted to the DCS. Communications must also be provided to the technical/analytical personnel who are responsible for analyzer maintenance, calibration, and quality assurance functions.

Information presented to the process operators on the DCS display screens should appear similar whether it comes from conventional process sensors (pressure, temperature, level, and flow transmitters) or from sophisticated on-line analyzers. The OLA data must also be available to the control procedures in the DCS for automatic process control. Process operators should be able to interrogate the OLA to confirm operational condition (calibration, QA requirements, etc.) and have the ability to start-up or shut-down the OLAs from the DCS operating console.

A prototype on-line analyzer to distributed control system network has been installed and is being tested at the Savannah River Laboratory. A block diagram of the existing prototype network is illustrated in Figure 1. Shown in Figure 2 is a conceptual online analyzer to distributed control system network for a production area application. 
Figure 1

\section{PROTOTYPE ON-LINE ANALYZER TO DCS NETWORK}

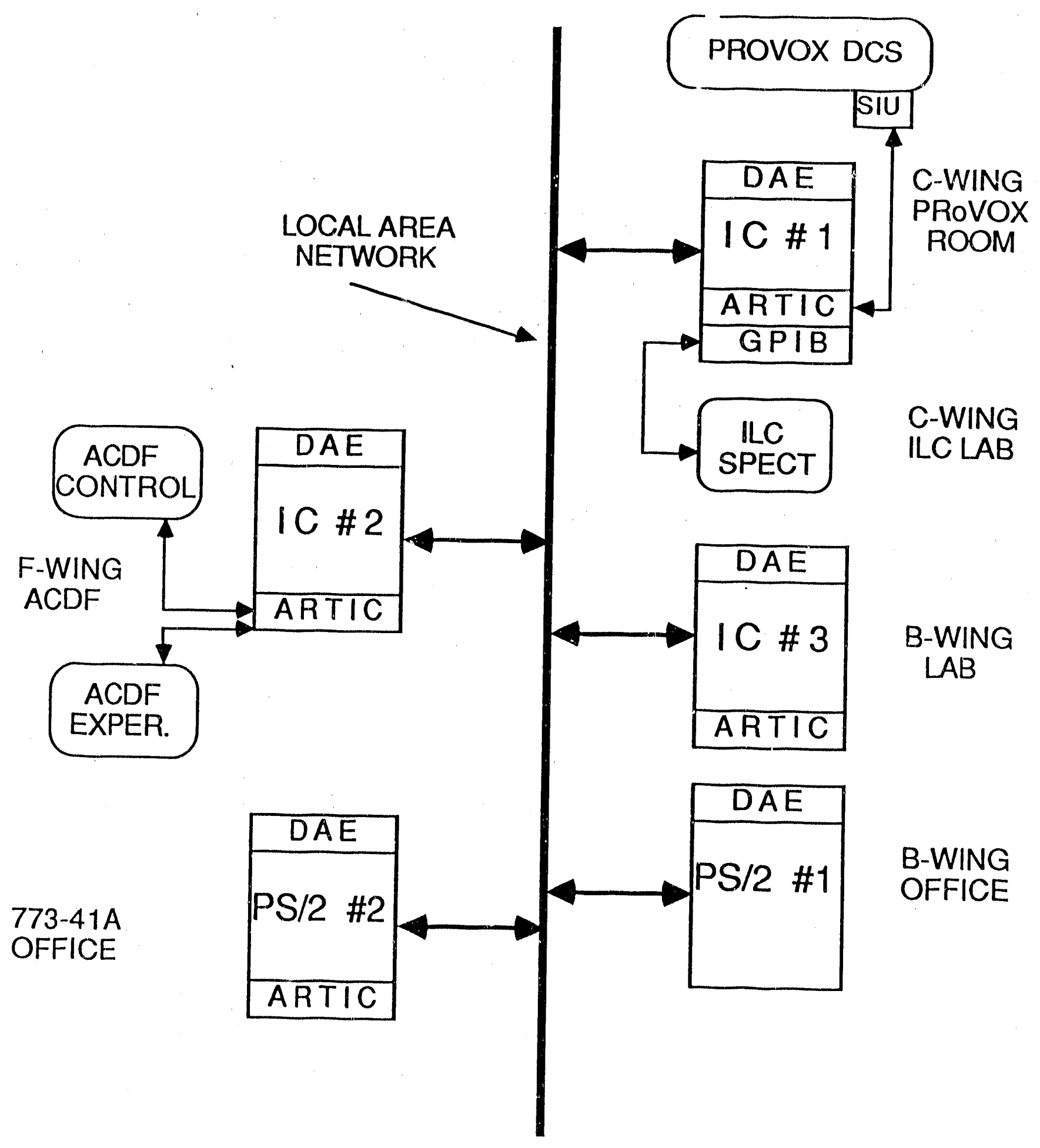




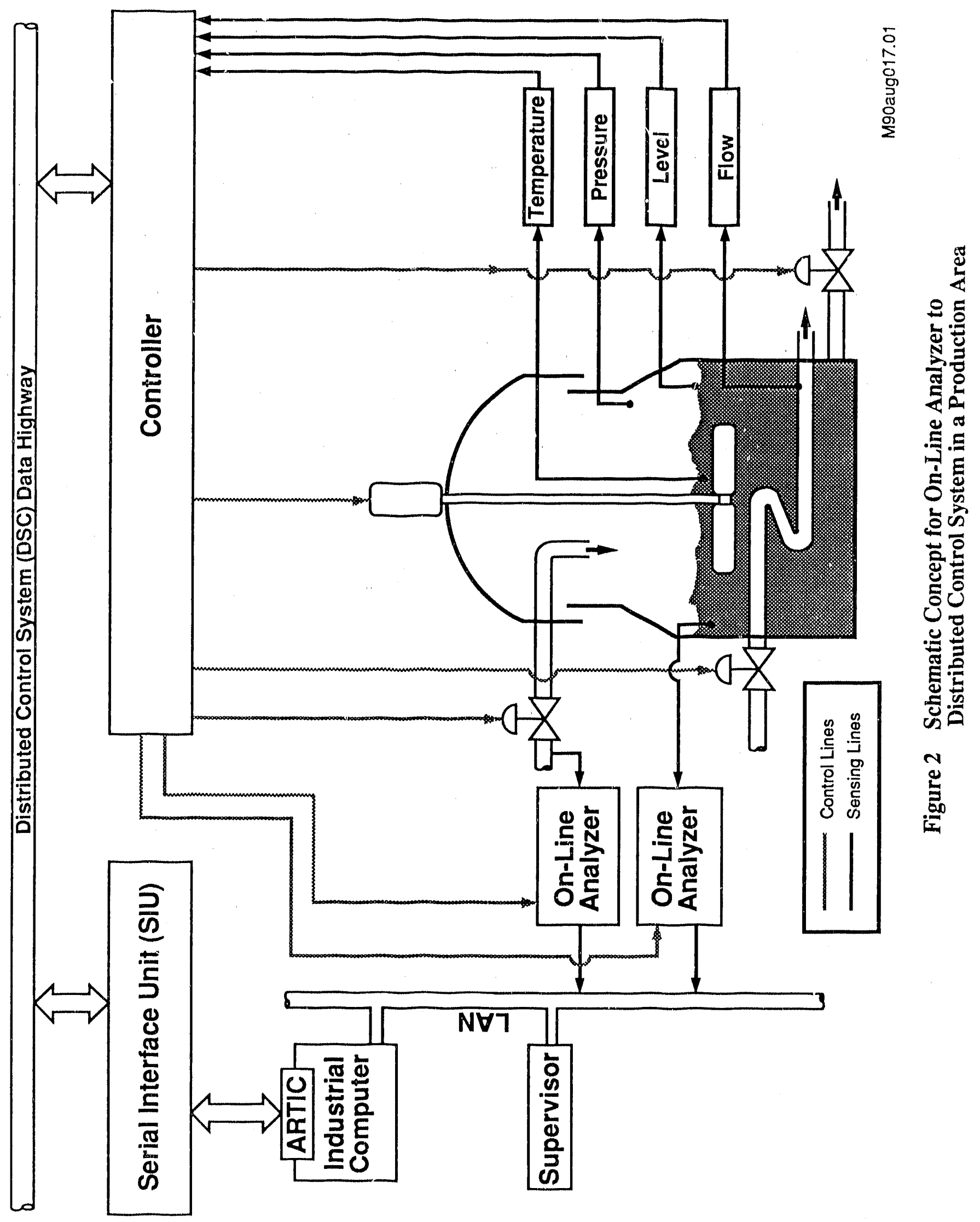




\section{DISCUSSION}

The principal components in the prototype on-line analyzer to distributed control system network being tested at the Savannah River Laboratory are:
1. An operating on-line analyzer
2. Personal computers in offices
3. Interconnection from the DCS data highway to an IBM industrial
computer
4. Interconnecting local area network
5. PC software to integrate the whole system.

\section{On-Line Analyzers}

A variety of analysis situations have been addressed with on-line analyzers. Whenever commercial instruments are available, they will be recommended. Examples include units to measure conductivity, density, oxidation-reduction potential, etc. Many situations cannot be solved with off-the-shelf instruments. In those instances, specialized analyzers must be developed. The computer platform for such analyzers will typically be either the IBM PC or Industrial Computer (a more rugged version of the PC, designed to withstand those environments typically found in production locations), or the DEC MicroVAX series. Chemical analyzers (e.g., spectrophotometers) will generally be designed around the IBM computer, while nuclear analyzers (e.g., gamma ray spectrometers) will use the DEC MicroVAX series. Part of the reason for the choice is the capability of the computers, but a great deal also depends on the analyzer designer and the computer system with which he is most familiar. Most chemists are comfortable programming in BASIC on IBM PC's.

When the analyzers are based on PCs or Industrial Computers, we have attempted to establish some hardware and software standards. SRS policy stipulates that IBM hardware will be used. For better real time response, we will use the IBM ARTIC coprocessor boards. We use OPTO-22 input/output hardware for interfacing of analyzer and sample systems to the PC/IC environment. Although most applications were developed using DOS, we are moving toward OS $/ 2$ for those applications requiring multi-tasking. Microsoft BASIC has been used extensively in the software development for existing OLA systems. It will continue to be used and adapted as appropriate for use in the newer operating systems such as OS/2. More and more frequently we can foresee a need to move toward the "C" language, not so much from the user advantage viewpoint, but because " $\mathrm{C}$ " is becoming the language of choice of systems developers and programmers. However, the Microsoft BASIC packages presently available allow " $\mathrm{C}$ " object code to be easily linked to BASIC. Therefore, at least for the near term, we will write drivers in " $\mathrm{C}$ " and continue using BASIC for the actual control programs.

Several options existed for the on-line analyzer to be used in this prototype network. The one chosen was a visible - ultraviolet spectrophotometer developed by ADS to monitor uranium or plutonium concentrations for solvent extraction separation studies, being carried out in a radiation shielded facility. Data from this analyzer, if readily available to the DCS used to control the experiments, would permit direct control of the miniature mixer-settler solvent extraction apparatus by the DCS. The actual analyzer that will be tested in this study is a Hewlett-Packard Diode Array Spectrophotometer (DAS). The instrument is capable of collecting a UV-visible spectrum from 190 to $820 \mathrm{~nm}$ in $2 \mathrm{~nm}$ increments. Acquisition time for a single spectrum is 0.1 second, using a General Purpose Interface Bus (GPIB) which is an IEEE-488 industry standard. Thus, measurements of a 
process can be accomplished in near real time. Because optical fibers are capable of trarismitting visible light over kilometer distances, the instrument can be coupled to several positions at varying distances from the instrument. This is especially attractive for a process instrument, as the instrument can be located in the control room under relatively benign environmental conditions.

The normal working range of the analyzer system, an HP 8452 DAS with optical fibers, is $300 \mathrm{~nm}$ to $820 \mathrm{~nm}$. The sensor in a DAS can be thought of as each individual unit of an array of photosensitive detectors, each illuminated by a different wavelength of light. Signals from all the detectors are combined to form a spectrum of 261 channels. These detectors are non-specific, meaning that the response at a particular wavelength is not necessarily related to one effect. Usually a response at a particular wavelength is the sum of all absorbing species in the system. For comparison a specific sensor would be a thermocouple which is responsive to only temperature. The problem with using a DAS instrument as a process sensor is that the spectra require an expert for interpretation. Thus, if the entire spectrum were to be sent to the DCS operator, it would be meaningless.

Fortunately, chemometrics ${ }^{\mathrm{a}}$ can be used to provide a meaningful number to the operator from the non-specific sensor. Using this technique the spectrum in Figure 3 can be reduced to a concentration value and an F-of-residuals test statistic ${ }^{b}$. These two number are now valuable pieces of information for the process control system.

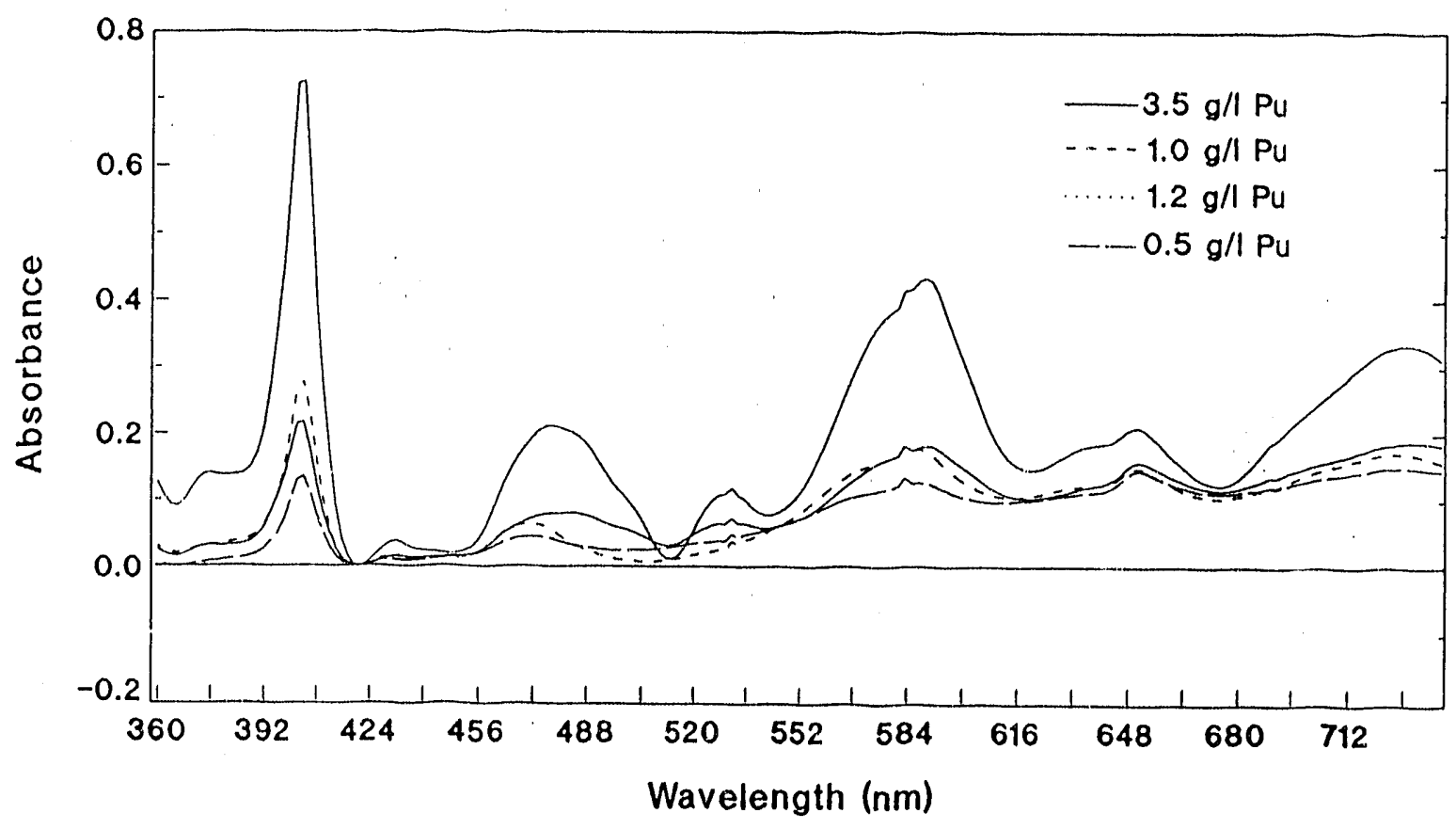

Figure 3 UV-Visible Spectr's

\footnotetext{
a Chemometrics is the application of statistical methods to chemical systems.

b The F-of-residuals is a measure of how much the sample "looks" like the calibration set.
} 


\section{Office PCs}

The prototype includes the connection of office personal cumputers to the network. This allows, for example, process development chemists to monitor the status of laboratory experiments, while analytical chemists monitor the status and operation of the OLA's on the network and participate in data interpretation. Doing this with the prototype network demonstates the OLA monitoring, calibration, and QA verification that would be done by Laboratories Department personnel for a Site OLA-DCS network.

The principal PC used for the prototype is an IBM PS/2 Model 80. (IBM PS/2 computers are one of the standard computers used at SRS. The Apple MacIntosh is another standard system.) This computer carries out the functions mentioned above, is used for software development, and is the file server for the prototype system.

This PC will be used to test and develop various applications under OS $/ 2$ which might be used by a scientist or engineer in the office environment. These applications could include integration of data into spreadsheets and presentations software, statistical data analysis, analyzer calibration, and various Quality Assurance functions. Commercially available software as well as in-house developed software is used for these applications.

\section{Distributed Control System (PRoVOX) to IBM Link}

The Savannah River Site supports a continuing program to upgrade the process control capabilities in their operating areas. The chemical separations areas have, for the last 10 years or so, been replacing manual control panels with modern, microprocessorbased distributed control systems. One of the distributed control systems used on the site is the Fisher Controls PRoVOX. Like most distributed control systems, the PRoVOX uses a dual data highway to interconnect controllers with operator consoles, etc. This coaxial cable data highway uses a proprietary data transfer protocol. Thus, transferring information from on-line analyzers to the data highways not a trivial matter.

Fisher Controls has recently announced a solution to transferring data to and from their PRoVOX data highway and a personal computer or industrial computer. The solution consists of a Serial Interface Unit (SIU), A Real-Time Interface Card (ARTIC) for the PC, and Computer Highway Interface Package (CHIP) software. The SIU provides a high speed serial (RS-422) port on the data highway. This port connects to the ARTIC board. The ARTIC card is a standard IBM product, available for AT-style or micro-channel architecture computers. CHIP consists of control and data base programs that run on the microprocessor in ARTIC, and allows communication with programs running in the PC. Versions of CHIP are available for MS/DOS and OS/2 operating systems. With this hardware and software, up to 500 points in the PRoVOX system can be made available in a data base residing on the ARTIC board. These data points are then accessible from t uth the PRoVOX and PC.

This provides an easy way for the DCS to control the on-line analyzers, and to transfer analyzer information to the DCS. The operations are transparent to the DCS operators. Information from the sophisticated on-line analyzers can be treated in much the same fashion as that from the more familiar pressure, temperature, level, and flow transmitters. 


\section{Local Area Network}

There are several choices for the computer network for this demonstration; both Ethernet and Token Ring Networks will be used.

The obvious choice is to use the existing Ethernet system. All laboratories and offices in the Technical Area at SRS are wired with connections to an Ethernet LAN, permitting PCs in any office or lab could be connected to the network without installing new cables. Most locations in the production areas also have Ethernet LAN connections. LANs in each site area are linked with a site-wide broad band network.

Two problems exist with the use of the Ethernet LAN. First, IBMs Distributed Automation Edition (DAE) requires OS/2 Extended Edition Version 1.2 to communicate via Ethernet. This software product has been announced, but is not yet available at SRS. Second, some of the information generated by on-line analyzers operating in SRS production areas is classified and should not be readily accessible throughout the site. This argues for either a small Ethernet LAN not connected with the rest of the site system, or a LÂN using a different protocol.

DAE presently supports the IBM Token Ring Network protocol. The use of Token Ring requires some additional wiring for the prototype networking demonstration in the laboratory. Interconnection PC's in office buildings adjacent to the laboratory building will be more difficult, but should be possible using an additional PC configured as a "bridge" from Token ring to Ethernet.

\section{Software}

The key to making this network work is the software that runs in each PC connected to the LAN. At the present time, there are several commercially available software products that probably would meet the needs of this task. When we were in the early stages of the task (early 1989), however, the choices were more limited. At that time, we identified two viable options for this software: Intellution (FIX and DMACS products) and IBM (DAE product). Both suppliers' products were evaluated, including vendor site visits, on-site training for the Intellution products, etc. The IBM solution was preferred and was purchased.

Programs have been written at the Savannah River Site to help in data interpretation, collect data from instruments, and to monitor sample positions unattended. These programs were developed using Microsoft's Quick BASIC Version 4.0 under DOS. Microsoft has just introduced a Professional BASIC Version 7.0 which can be used to recompile programs to operate under OS $/ 2$. The BASIC Version 7.0 can make mixed language calls, which should allow the coupling of the present program to the DAE program.

The IBM Distributed Automation Edition (DAE) is a data communications system for managing data and device communications on a computer network. It consists of software running on all computers within a network to allow them to share data and to control various devices (such as PC-based on-line analyzers) attached to the network. DAE provides a uniform interface to the data and devices and provides a common software platform for application programming development. It is very complex and not particularly friendly in its user interface. Hence, 3-4 weeks of training courses, which in some ways only begin to scratch the surface in terms of user/programmer training. 
For DAE to run on Industrial Computers and PS/2 Personal Computers (PCs) requires the OS/2 Extended Edition operating system. It will not run under DOS. IBM also highly recommends the use of their Micro Channel Architecture (MCA) to provide reasonable speed in operations.

IBM announced another software package in early 1990. This software, called PlantWorks, is an application enabler program which uses a high level programming language. The programmer constructs his application as a flow chart in the editor. The program then converts the flowchart to " $\mathrm{C}$ " and compiles it to an executable program. This program has been ordered, and will be integrated into our system upon receipt.

An advantage of the DAE and PlantWorks software packages from IBM is that IBM will support the programs. DAE and PlantWorks are supported through IBM's Systems Application Architecture (SAA) which is a guarantee from IBM that the software and hardware will be protected through use of standard, migratable products.

\section{SUMMARY}

Our normal procedure for introducing a new analyzer or concept such as the on-line analyzer to DCS networking method to the operating areas at SRS is to provide a demonstration on a small scale system. The Analytical Development Section of SRL is establishing such a demonstration on laboratory scale chemical processes. The test system uses an IBM Industrial Computer to operate a Hewlett-Packard Diode Array

Spectrophotometer and send information to a Fisher Controls PRoVOX Distributed Control System. With a successful demonstration of the analyzer to DCS networking system, operating personnel are much more likely to accept analyzers to help control their processes. This will enhance productivity and safety at the Savannah River Site. 


\section{OBJECTIVE:}

To develop and 'nstall lntegrated systems of automated samplers, on-line process analyzers, and process control methods

using the best approprlate technology

and

prloritizte to meet the needs of Operating Departments.

GOALS OF ON-LINE ANALYZER tO DCS COMMUNICATIONS:

- Information Transparent to DCS Operator

- Control OLA from DCS

- Verify Proper OLA Operation from DCS and from Lab 


\section{COMPONENTS OF DEMONSTRATION LAN}

- On-Line Analyzer(s)

- Office PC's

- PRovox to IBM Link

- Local Area Network

- Software

ON-LINE ANALYZERS

- Variety of Types

- Based on Industrial Computers

- ' $C$ ' Programming Language

- ARTIC Boards

- i/O to Analyzer Hardware 


\section{OFFICE COMPUTERS}

- "Site Standard" PC̈s

- Monitor OLA Status

- Data Handling

- File Server

IDCS IO LAN LINK

- Fisher PRoVOX DCS

- Serial Interface Unit

- ARTIC Bcard

- Chip Software 
LOCAL AREA NIETWORK

- Existing ETHERNET

- NeW TOKEN RING

SOFTWARE

- IBM CIM Packages

- Distributed Automation

Edition (DAE)

- PlantWorks 

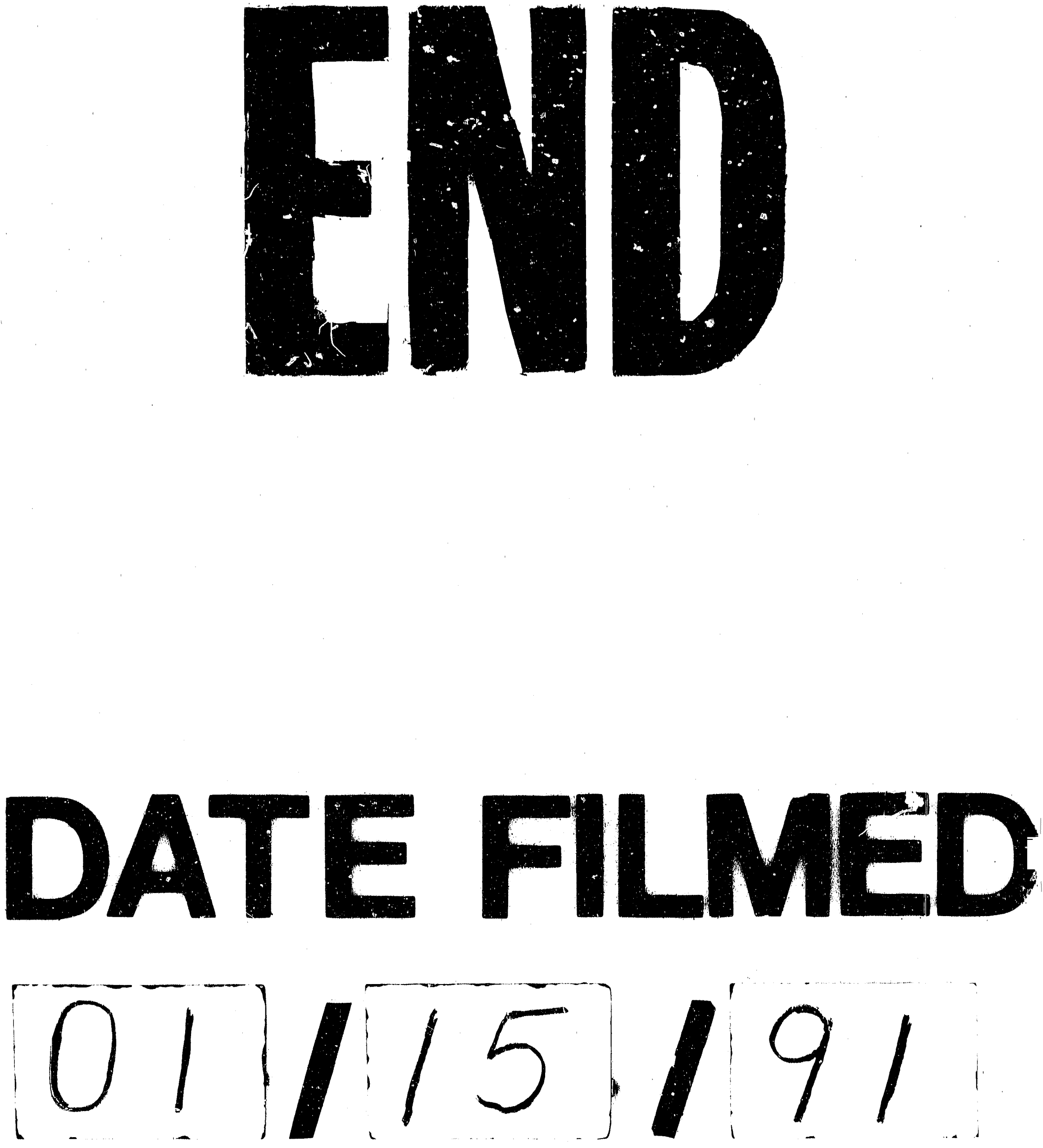
